\title{
A TOPOLOGICAL APPROACH TO EXTREME POINTS IN FUNCTION SPACES
}

\author{
JOHN CANTWELL ${ }^{1}$
}

1. Introduction. Let $X$ be a Hausdorff space. Denote by $C^{n}(X)$ the space of all $R^{n}$-valued, bounded, continuous functions on $X$, with the usual supremum norm. If $X$ is compact it is known that the closed unit ball in $C^{1}(X)$ is the closed convex hull of its extreme points if and only if $X$ is totally disconnected (see Goodner [2]). If $X$ is compact, Phelps [6] has proved that the closed unit ball in $C^{2}(X)$ is the closed convex hull of its extreme points. If $X$ is compact metric Peck [5] has proved that the closed unit ball in $C^{2}(X)$ is the convex hull of its extreme points if and only if $\operatorname{dim} X \leqq 1$.

The proofs given by Phelps [6] and Peck [5] rely on measure theory. We remove the measure-theoretic aspects of their arguments and obtain theorems valid for a broader class of spaces $X$, and, in the case of the result of Peck, for arbitrary $n$. We prove:

Theorem I. Let $X$ be a Hausdorff space. The closed unit ball of $C^{2}(X)$ is the closed convex hull of its extreme points.

Theorem II. Let $X$ be a normal Hausdorff space. For $n=2,3, \cdots$ the following two statements are equivalent:

(i) $\operatorname{dim} X \leqq n-1$,

(ii) the closed unit ball in $C^{n}(X)$ is the convex hull of its extreme points.

The proof of Theorem I is elementary. Theorem II depends on some deep results of dimension theory. We remark that Theorem I is true with $C^{2}(X)$ replaced by $C^{n}(X)$ for any $n \geqq 2$. One can see this by using the method of the proof of Lemma 2 to prove a modified Lemma 1. If one replaces " 2 " by " $n$ " in Lemma 1 , one no longer has a continuous unit vector field on $S^{n-1}$ to work with if $n$ is odd. One must therefore use the method of Lemma 2 to obtain $f$ as a convex combination of four functions, instead of two functions, with the desired properties. The proof of Theorem I (with " 2 " replaced by “ $n$," $n \geqq 2$ ) then follows as before from this modified Lemma 1 .

2. Notation and preliminaries. We let $D^{n}$ (respectively $S^{n-1}$ ) denote the closed unit disc (respectively the unit sphere) in $R^{n}$. Let $N_{\epsilon}(x)$

Received by the editors April 4, 1967.

${ }^{1}$ Supported in part by NSF Grant GP-5337. 
$=\left\{y \in R^{n}|| y-x \mid<\epsilon\right\}$. All functions considered in this paper are continuous. The closed unit ball in $C^{n}(X)$, denoted by $U^{n}(X)$, consists of those $f \in C^{n}(X)$ such that $f(X) \subset D^{n}$. It is easy to see that the set of extreme points of $U^{n}(X)$, denoted by $E^{n}(X)$, consists of those $f \in C^{n}(X)$ such that $f(X) \subset S^{n-1}$. By $\operatorname{dim} X$ we mean the Lebesgue covering dimension.

We make use of the following theorems from dimension theory.

Theorem A. Let $X$ be a normal space. $\operatorname{dim} X \leqq n$ if and only if for each closed subset $C$ of $X$ and each map from $C$ into $S^{n}, f$ has an extension $f^{*}$ from $X$ into $S^{n}$.

Theorem B. If $X$ is a normal space, $\operatorname{dim} X \leqq n-1,1 \geqq \delta>0$, and $f$ maps $X$ into $D^{n}$, then there exists a map $g$ from $X$ into $D^{n}$ such that

$$
g(x)=f(x) \text { if }|f(x)| \geqq \delta,|g(x)|=\delta \text { if }|f(x)| \leqq \delta .
$$

The reader is referred to Dowker [1] or Hemmingsen [3] for Theorem A. The reader is also referred to Nagata [4, Theorem III.2, p. 53], for Theorem $A$ in the special case $X$ is metric.

Theorem B follows rather easily from Theorem A. In fact, if $f$ maps $X$ into $D^{n}$, define $g$ as follows. Let $S_{\delta}^{n-1}$ (respectively $D_{\delta}^{n}$ ) denote the sphere (respectively closed ball) of radius $\delta$ about the origin in $R^{n}$. Then $f^{-1}\left(D_{\delta}^{n}\right)$ is closed in $X$ and thus $\operatorname{dim} f^{-1}\left(D_{\delta}^{n}\right) \leqq n-1$. Also, $f^{-1}\left(S_{\delta}^{n-1}\right)$ is closed in $f^{-1}\left(D_{\delta}^{n}\right)$. Thus by Theorem $\mathrm{A}, f \mid f^{-1}\left(S_{\delta}^{n-1}\right)$ can be extended to a map $f^{*}$ from $f^{-1}\left(D_{\delta}^{n}\right)$ to $S_{\delta}^{n-1}$. Let

$$
g(x)=f(x) \text { if }|f(x)| \geqq \delta, \quad g(x)=f^{*}(x) \text { if }|f(x)| \leqq \delta .
$$

3. Proof of Theorem I. We need an easy lemma.

Lemma 1. If $f$ maps $X$ to $D^{2}$ and $y \notin f(X)$ for some $y \in D^{2}$ with $0 \leqq|y|<1$, then there exist $g$ and $h$ mapping $X$ to $D^{2}-N_{1-|y|}(y)$ such that $f=\frac{1}{2}(g+h)$.

Proof. Let $v(x)$ be a continuous unit vector field on $S^{1}$. If $f(x)$ $\notin N_{1-|y|}(y)$ set $g(x)=h(x)=f(x)$. If $f(x) \in N_{1-|y|}(y)$ set

$$
\begin{aligned}
& g(x)=f(x)+\lambda(x) v(f(x)-y /|f(x)-y|), \\
& h(x)=f(x)-\lambda(x) v(f(x)-y /|f(x)-y|),
\end{aligned}
$$

where $\lambda(x)=\left((1-|y|)^{2}-|f(x)-y|^{2}\right)^{1 / 2}$. Clearly $f=\frac{1}{2}(g+h)$. A calculation shows $g$ and $h$ map $X$ into $D^{2}-N_{1-|y|}(y)$.

To prove Theorem I let $f$ map $X$ to $D^{2}$ and let $\epsilon>0$ be given. Let $f^{*}$ be a map from $X$ to $D^{2}-N_{\epsilon}(1,0)$ such that $d\left(f, f^{*}\right) \leqq \epsilon$. Let $y_{0}, \cdots, y_{m} \in D^{2}$ be a sequence of points such that $y_{0}=(1,0), y_{m}$ 
$=(0,0), 0<d\left(y_{1}, y_{0}\right)<\epsilon$, and $d\left(y_{i+1}, y_{i}\right)<1-\left|y_{i}\right|, i=1, \cdots, m-1$. Now apply Lemma $1, m$ times. First $y_{1} \notin f^{*}(X)$. Therefore $f^{*}=\frac{1}{2}(g+h)$ where $y_{2} \notin g(X) \cup h(X)$. We then apply Lemma 1 to $g$ and $h$ to get $g=\frac{1}{2}\left(g^{*}+h^{*}\right), \quad h=\frac{1}{2}\left(g^{* *}+h^{* *}\right) \quad$ with $\quad y_{3} \notin g^{*}(X) \cup h^{*}(X)$ $\cup g^{* *}(X) \cup h^{* *}(X)$. The $m$ th application gives us $2^{m}$ functions $g_{i}$ from $X$ to $S^{1}$ such that $f^{*}=1 / 2^{m} \sum g_{i}$. Theorem $I$ is proved.

4. Proof of Theorem II. We first prove a lemma.

Lemma 2. If $f \in U^{n}(X), n=2,3, \cdots$, and $0 \notin f(X)$ then there exist $g, h, g^{*}, h^{*} \in E^{n}(X)$ such that $f=(1 / 4)\left(g+h+g^{*}+h^{*}\right)$.

Proof. We remark that if $(n-1)$ is odd there is a continuous unit tangent vector field on $S^{n-1}$ and the method of Lemma 1 works. In that case we get $f=\frac{1}{2}(g+h)$ with $g, h \in E^{n}(X)$. For arbitrary $n$ we proceed as follows. Let $\sigma=(0, \cdots, 0,-1)$ be the south pole of $S^{n-1}$. Let $D_{+}^{n}=\left\{\left(y_{1}, \cdots, y_{n}\right) \in D^{n} \mid y_{n} \geqq 0\right\}$. Let $v=\left(v_{1}, \cdots, v_{n}\right)$ be a continuous field of unit tangent vectors on $S^{n-1}-\{\sigma\}$. Let $\lambda$ be a continuous map from $D^{n}-\{0\}$ to $[0,1]$ such that:

$$
\lambda(y)=1 \text { if }-y_{n} \leqq|y|\left(1-|y|^{2}\right)^{1 / 2}, \lambda(y)=0 \text { if }-y_{n}=|y| .
$$

Define

$$
\begin{aligned}
& g^{\prime}(x)=f(x)+\lambda(f(x))\left(1-|f(x)|^{2}\right)^{1 / 2} v(f(x) /|f(x)|) \\
& g^{\prime}(x)=f(x) \quad \text { if }-f_{n}(x)=|f(x)| . \\
& h^{\prime}(x)=f(x)-\lambda(f(x))\left(1-|f(x)|^{2}\right)^{1 / 2} v(f(x) /|f(x)|) \\
& h^{\prime}(x)=f(x) \quad \text { if }-f_{n}(x)=|f(x)| .
\end{aligned}
$$

Clearly $g^{\prime}$ and $h^{\prime}$ are continuous and $f=\frac{1}{2}\left(g^{\prime}+h^{\prime}\right)$.

Further

$$
\begin{aligned}
\left|g^{\prime}(x)\right| & =\left|f(x)+\lambda(f(x))\left(1-|f(x)|^{2}\right)^{1 / 2} v(f(x) /|f(x)|)\right| \\
& =\left(|f(x)|^{2}+\lambda(f(x))^{2}\left(1-|f(x)|^{2}\right)\right)^{1 / 2} \leqq 1
\end{aligned}
$$

This follows since $f(x)$ and $v(f(x) /|f(x)|)$ are orthogonal and $v(y)$ is a unit vector. Thus $g^{\prime}(X) \subset D^{n}$. Similarly, $h^{\prime}(X) \subset D^{n}$.

If $-f_{n}(x) \leqq|f(x)|\left(1-|f(x)|^{2}\right)^{1 / 2}$ then $\lambda(f(x))=1$ so the above argument gives $\left|g^{\prime}(x)\right|=1$. If $-f_{n}(x)>|f(x)|\left(1-|f(x)|^{2}\right)^{1 / 2}$ then $v_{n}(f(x) /|f(x)|) \leqq f(x)$. In fact if $v_{n}(f(x) /|f(x)|)>|f(x)|$ under the assumed condition on $f_{n}(x)$, obtain a contradiction by using the fact that the inner product of $f(x)$ with $v(f(x) /|f(x)|)$ is zero and by estimating the first $n-1$ terms and the last term of this inner product 
separately, using Cauchy's inequality for the first $n-1$ terms. Therefore $g_{n}^{\prime}(x)=f_{n}(x)+\lambda(f(x))\left(1-|f(x)|^{2}\right)^{1 / 2} v_{n}(f(x) /|f(x)|) \leqq f_{n}(x)$ $+\left(1-|f(x)|^{2}\right)^{1 / 2}|f(x)|<f_{n}(x)-f_{n}(x)=0$. Therefore in either case $g^{\prime}(X) \cap D_{+}^{n} \subset S^{n-1}$. Similarly, $h^{\prime}(X) \cap D_{+}^{n} \subset S^{n-1}$.

To prove Lemma 2 one now applies the above process to the functions $-g^{\prime}$ and $-h^{\prime}$ to get $g^{\prime}=\frac{1}{2}(g+h), h^{\prime}=\frac{1}{2}\left(g^{*}+h^{*}\right)$ with $g(X) \cup h(X) \cup g^{*}(X) \cup h^{*}(X) \subset S^{n-1}$.

To prove Theorem II, suppose $\operatorname{dim} X \leqq n-1$. Theorem B implies there is a continuous function $g^{*}$ from $X$ to $D^{n}$ such that:

$$
\begin{aligned}
g^{*}(x) & =f(x) & \text { if }|f(x)| & \geqq 1 / 3, \\
\left|g^{*}(x)\right| & =1 / 3 & & \text { if }|f(x)| \leqq 1 / 3 .
\end{aligned}
$$

Put $h^{*}=2 f-g^{*}$. Clearly $g^{*}, h^{*} \in U^{n}(X)$. As above, there exists a continuous function $h$ from $X$ to $D^{n}$ such that:

$$
\begin{array}{rlrl}
h(x) & =h^{*}(x) & & \text { if }\left|h^{*}(x)\right| \geqq 1 / 9, \\
|h(x)| & =1 / 9 & \text { if }\left|h^{*}(x)\right|<1 / 9 .
\end{array}
$$

Put $g=2 f-h$. Clearly $g, h \in U^{n}(X), f=\frac{1}{2}(g+h)$, and $0 \notin h(X)$. Further, since:

$$
\left|g(x)-g^{*}(x)\right|=\left|h(x)-h^{*}(x)\right| \leqq 2 / 9
$$

and $\left|g^{*}(x)\right| \geqq 1 / 3$ for all $x \in X$ it follows that $0 \notin g(X)$. We now apply Lemma 2 to $g$ and $h$ to obtain $f$ as a convex combination of eight elements of $E^{n}(X)$.

Conversely, suppose $U^{n}(X)$ is the convex hull of $E^{n}(X)$. We want to show $\operatorname{dim} X \leqq n-1$. By Theorem A, it suffices to show that for any closed subset $C$ of $X$ and any map $f$ from $C$ to $S^{n-1}, f$ can be extended to a map $g$ from $X$ to $S^{n-1}$. Suppose $f$ and $C$ are given as above. By Tietze's extension theorem $f$ has an extension $f^{*}$ from $X$ to $D^{n}$, i.e. $f^{*} \in U^{n}(X)$. By assumption there exist $f_{1}, \cdots, f_{k} \in E^{n}(X)$ and $r_{1}, \cdots, r_{k} \in R$ such that $f^{*}=\sum r_{i} f_{i}, \sum r_{i}=1$, and $r_{1}, \cdots, r_{k} \geqq 0$. For $i=1, \cdots, k, f_{i} \in E^{n}(X)$ so $f_{i}$ maps $X$ to $S^{n-1}$. Further, if for some $x \in C$, and some $j \in\{1, \cdots, k\}, f(x) \neq f_{j}(x)$ then by the triangle inequality $1=|f(x)|=\left|\sum r_{i} f_{i}(x)\right|<\sum\left|r_{i} f_{i}(x)\right|=\sum r_{i}=1$. Thus, for each $j \in\{1, \cdots, k\}$, and each $x \in C, f(x)=f_{j}(x)$. Thus each $f_{j}, j=1, \cdots, k$, gives the desired extension of $f$ and Theorem II is proved.

5. It is interesting to note that we have actually proved that if $\operatorname{dim} X \leqq n-1$ any element of $U^{n}(X)$ is the convex combination of eight elements of $E^{n}(X)$. If $(n-1)$ is odd the number eight can be 
replaced by four. It seems that one should be able to improve on these numbers.

\section{REFERENCES}

1. C. H. Dowker, Mapping theorems for non-compact spaces, Amer. J. Math. 69 (1947), 200-242.

2. D. B. Goodner, The closed convex hull of certain extreme points, Proc. Amer. Math. Soc. 15 (1964), 256-258.

3. E. Hemmingsen, Some theorems in dimension theory for normal Hausdorff spaces, Duke Math. J. 13 (1946), 495-504.

4. J. Nagata, Modern dimension theory, Interscience, New York, 1965.

5. N. T. Peck, Representation of functions in $C(X)$ by means of extreme points, Proc. Amer. Math. Soc. 18 (1967), 133-135.

6. R. R. Phelps, Extreme points in function algebras, Duke Math. J. 32 (1965), 267-277.

UNIVERSITY OF IOWA 